\title{
Rectification in Spin-Orbit Materials Using Low-Energy-Barrier Magnets
}

\author{
Shehrin Sayed,,${ }^{1,2,{ }^{*}}$ Kerem Y. Camsari, ${ }^{1}$ Rafatul Faria, ${ }^{1}$ and Supriyo Datta ${ }^{1}$ \\ ${ }^{1}$ Electrical and Computer Engineering, Purdue University, West Lafayette, Indiana 47907, USA \\ ${ }^{2}$ Electrical Engineering and Computer Science, University of California, Berkeley, California 94720, USA
}

(Received 16 November 2018; revised manuscript received 1 April 2019; published 23 May 2019)

\begin{abstract}
The coupling of spin-orbit materials to high-energy-barrier (approximately 40-60 $k_{B} T$ ) nanomagnets has recently attracted growing interest due to exciting new physics and various spintronic applications. We predict that a coupling between the spin-momentum locking (SML) observed in spin-orbit materials and low-energy-barrier magnets (LBMs) should exhibit a unique multiterminal rectification for arbitrarily small-amplitude channel currents. The basic idea is to measure the charge-current-induced spin accumulation in the SML channel in the form of a magnetization-dependent voltage using an LBM, either with an in-plane or perpendicular anisotropy (IMA or PMA, respectively). The LBM feels an instantaneous spin-orbit torque due to the accumulated spins in the channel, which causes the average magnetization to follow the current, leading to the nonlinear rectification. We discuss the frequency band of this multiterminal rectification, which can be understood in terms of the angular-momentum conservation in the LBM. For a fixed spin-current from the SML channel, the frequency band is same for LBMs with IMA and PMA, as long as they have the same total magnetic moment in a given volume. The proposed all-metallic structure could find application as a highly sensitive passive radio-frequency (rf) detector and as an energy harvester from weak ambient sources, where standard technologies may not operate.
\end{abstract}

DOI: 10.1103/PhysRevApplied.11.054063

\section{INTRODUCTION}

The interplay between spin-orbit materials and nanomagnetism has attracted much attention due to interesting phenomena, e.g., spin-orbit torque switching [1,2], probing of the spin-momentum locking [3-8], spin amplification [9], the spin battery [10], and skyrmion dynamics [11,12], among other examples. In this paper, we predict that the spin-momentum locking (SML) observed in spin-orbit materials when coupled to a nanomagnet with a low energy barrier will rectify the channel current in the form of a voltage in a multiterminal structure. We start our arguments with the spin-potentiometric measurements that are well established in diverse classes of spin-orbit materials (see, e.g., [3-8]), where a high-energy-barrier stable ferromagnet (FM) is used to measure the charge-current-induced spin potential in the SML channel. We show that such spinpotential measurement on a metallic SML channel using a low-energy-barrier magnet (LBM) will result in a rectified voltage, even for an arbitrarily small channel current.

\footnotetext{
*ssayed@berkeley.edu
}

Published by the American Physical Society under the terms of the Creative Commons Attribution 4.0 International license. Further distribution of this work must maintain attribution to the author(s) and the published article's title, journal citation, and DOI.
The discussions on multiterminal rectification are limited to the linear response regime of transport in the SML channel and the nonlinearity occurs due to the spin-orbittorque- (SOT) driven magnetization dynamics of the LBM. We further show that the multiterminal rectification is limited by a characteristic frequency of the LBM that can be understood in terms of angular-momentum conservation between the spins injected from spin-orbit materials and the spins absorbed by the LBM. We argue that, for a fixed spin-current from the SML channel, the characteristic frequency is the same for LBMs with in-plane and perpendicular magnetic anisotropies (IMA and PMA, respectively), as long as they have the same total magnetic moment in a given volume.

We analyze the rectification in the proposed all-metallic structure [see Fig. 1(a)] considering both IMA and PMA LBMs and provide simple models to understand the underlying mechanisms of (i) the SOT-induced magnetization pinning and (ii) the frequency band of the rectification. We compare the simple models with detailed numerical simulations using an experimentally benchmarked multiphysics framework [13]. The simulations are carried out using a transmission-line model for the SML channel [14] and a stochastic Landau-Lifshitz-Gilbert (s-LLG) model for LBM [15], considering thermal noise within the magnet. We consider the spin-polarization axis to be in the plane of the SML channel and orthogonal to the current 

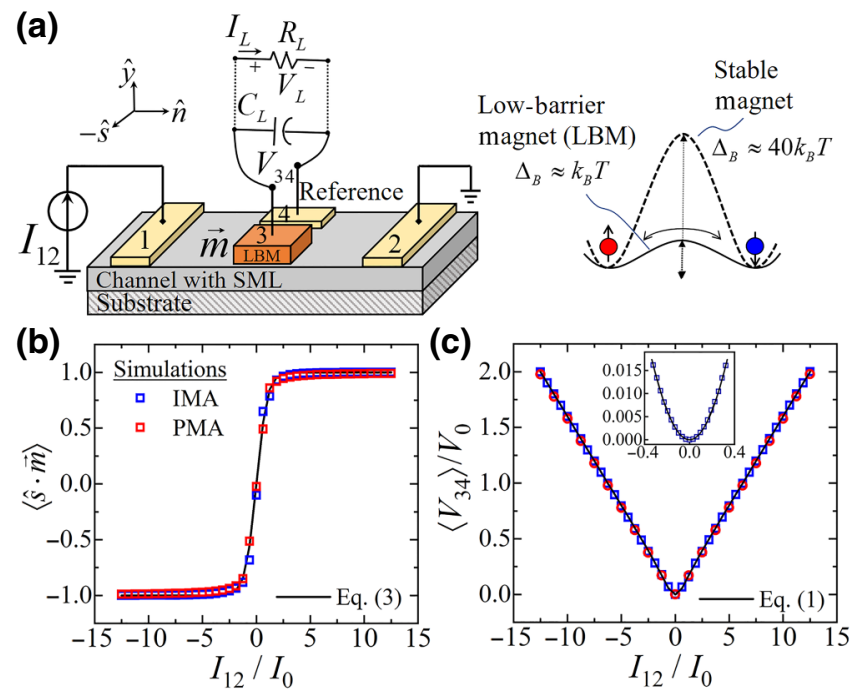

FIG. 1. (a) A multiterminal structure with a LBM on top of a channel with SML. The LBM can have in-plane or perpendicular anisotropies (IMA or PMA). (b) The average magnetization $\langle\hat{s}$. $\vec{m}\rangle$ of the LBM. (c) The average voltage $\left\langle V_{34}\right\rangle$ between the LBM and a reference contact, as a function of the input current $I_{12}$. The inset shows an enlarged version of $\left\langle V_{34}\right\rangle$ for a very small input current, which exhibits a parabolic nature. The simulations are compared using Eqs. (1) and (3). The $I_{0}$ values for IMA and PMA are $80 \mu \mathrm{A}$ and $1.6 \mu \mathrm{A}$, respectively. Here, $V_{0}=I_{0} R_{B}$.

flow direction. Hence, in the present discussion, pinning for a IMA or a PMA magnet occurs along the easy axis or the hard axis, respectively.

We argue that such wide-band rectification in an allmetallic structure [Fig. 1(a)] could be used for "passive" radio-frequency (rf) detection. Recently, magnetic-tunneljunction (MTJ) diodes with a stable magnet as the free layer and under an external dc-current bias have demonstrated a sensitivity that is several orders of magnitude higher compared to that of state-of-the-art Schottky diodes [16-18]. However, the reported no-bias sensitivity is lower or comparable to that of semiconductor diodes. The lowbarrier nature of the magnet in the proposed structure should exhibit a no-bias sensitivity as high as those observed using state-of-the-art technologies under external biases [16-18]. Furthermore, we discuss the possibility of harvesting energy from weak ambient sources, where standard technologies may not operate.

The paper is organized as follows. In Sec. II, we establish the concept of the multiterminal rectification in the SML channel using LBM, starting from the wellestablished spin-potentiometric measurements typically carried out with high-energy-barrier stable magnets. In Sec. III, we discuss the frequency bandwidth of the rectification and provide a simple model that applies to LBMs with both IMA and PMA. We argue, using detailed simulation results, that such bandwidth arises due to the principles of angular-momentum conservation between the spins injected from the SML channel and the spins absorbed by the LBM. In Sec. IV, we discuss possible applications of the proposed all-metallic structure in "passive" rf detection and energy harvesting. We argue that the no-bias sensitivity value of the proposed rectifier can be as high as those observed in state-of-the-art technologies under external bias. Finally, in Sec. V, we end with a brief conclusion.

\section{MULTITERMINAL RECTIFICATION}

We start our arguments with the well-established spin-potentiometric measurements [3-8], where the chargecurrent-induced spin potential in the SML channel is measured in the form of a magnetization-dependent voltage using a stable FM. The voltage at the FM with respect to a reference normal-metal (NM) contact, placed at the same position along the current path as the FM [see Fig. 1(a)], is given by $[19,20]$

$$
V_{34}(\vec{m})=(\hat{s} \cdot \vec{m}) \frac{\alpha \xi p_{0} p_{f} R_{B}}{2} I_{12}
$$

which shows opposite signs for the two magnetic states of the FM under a fixed channel current $I_{12}$ flowing along the $\hat{n}$ direction [see Fig. 1(a)]. Here, $\hat{s}$ is the spin-polarization axis in the SML channel defined by $\hat{y} \times \hat{n}$, where $\hat{y}$ is the out-of-plane direction [14], $\vec{m}$ is the magnetization vector, $p_{f}$ is the FM polarization, $0 \leq \xi \leq 1$ is the currentshunting factor [21] of the contact, with 0 and 1 indicating very high and very low shunting, respectively, $p_{0}$ is the degree of SML in the channel [14], $\alpha \approx 2 / \pi$ is an angular averaging factor [14], and $R_{B}=\left(h / q^{2}\right)\left(1 / M_{t}\right)$ is the ballistic resistance of the channel with total number of modes $M_{t}$ ( $q$, electron charge; $h$, Planck's constant).

Note that Eq. (1) is valid all the way from the ballistic to the diffusive regime of operation $[14,19]$. We restrict our discussion to the linear response, where $V_{34}(\vec{m})$ in Eq. (1) scales linearly with $I_{12}$ and satisfies the Onsager reciprocity relation $[21,22]$

$$
R_{i j, k l}(\vec{m})=R_{k l, i j}(-\vec{m}),
$$

with $R_{i j, k l}=V_{k l} / I_{i j}$. Onsager reciprocity does not require any specific relation between $R_{i j, k l}(\vec{m})$ and $R_{i j, k l}(-\vec{m})$ in the linear-response regime and the phenomenon described by Eq. (1) has been observed on diverse spin-orbit materials, e.g., topological insulators (TIs) [3-6], Kondo insulators [23], transition metals [7], semimetals [24], and semiconductors [8].

To measure Eq. (1) from a highly resistive SML channel (e.g., a TI [3-6], a semiconductor [8], etc.) using a metallic FM, a thin tunnel barrier is usually inserted at the interface. This tunnel barrier effectively enhances $V_{34}$ by improving $\xi$ [21]; however, it degrades the spin injection into the FM from the SML channel. It has recently been 
demonstrated [7] that $V_{34}$ can be measured with a metallic FM in direct contact with metallic SML channels (e.g., $\mathrm{Pt}, \mathrm{Ta}, \mathrm{W}$, etc.), which indicates the possibility of spinvoltage reading (e.g., [7]) and SOT writing (e.g., [1,2]) of the nanomagnet within same setup with different current magnitudes [20].

The energy barrier of a monodomain magnet is given by $\Delta_{B}=\frac{1}{2} H_{k} M_{s} \Omega$ [25], where $H_{k}$ is the anisotropy field, $M_{s}$ is the saturation magnetization, and $\Omega$ is the FM volume. For a stable FM, $\Delta_{B} \approx 40 \sim 60 k_{B} T$ and exhibits the very long retention time $\tau \propto \exp \left(\Delta_{B} / k_{B} T\right)$ of the magnetization state ( $k_{B}$, Boltzmann constant; $T$, temperature). LBMs have very small $\tau$ values and the $\hat{s} \cdot \vec{m}$ component becomes random within the range $\{+1,-1\}$ driven by the thermal noise. Experimentally, LBMs have been achieved by lowering the total moment $\left(M_{s} \Omega\right)$ [26] or by lowering the anisotropy field $\left(H_{k}\right)$, either by increasing the thickness of a PMA [27] or by making a circular IMA with no shape anisotropy [28].

At equilibrium $\left(I_{12}=0\right)$, the time-averaged $\langle\hat{s} \cdot \vec{m}\rangle=0$ for an LBM. For $I_{12} \neq 0$, induced nonequilibrium spins in the channel apply SOT on the LBM and $\langle\hat{s} \cdot \vec{m}\rangle$ follows the accumulated spins, which can be calculated using

$$
\langle\hat{s} \cdot \vec{m}\rangle=\frac{\int_{\phi=-\pi}^{\phi=\pi} \int_{\theta=0}^{\theta=\pi}(\hat{s} \cdot \vec{m}) \rho \sin \theta d \theta d \phi}{\int_{\phi=-\pi}^{\phi=\pi} \int_{\theta=0}^{\theta=\pi} \rho \sin \theta d \theta d \phi},
$$

where $\rho$ is the probability distribution function of the magnetization of the LBM under a particular $I_{12}$, which can be obtained from the Fokker-Planck equation $[29,30]$. The dependence of $\langle\hat{s} \cdot \vec{m}\rangle$ on $I_{12}$, deduced from Eq. (2) for a particular LBM, can in principle be any saturating odd functions, e.g., the Langevin function for a low-barrier PMA (see Appendix A).

We approximate $\langle\hat{s} \cdot \vec{m}\rangle$ in Eq. (2) with a tanh functional dependence on $I_{12}$, given by

$$
\langle\hat{s} \cdot \vec{m}\rangle \approx \tanh \left(\frac{I_{12}}{I_{0}}\right),
$$

which is in good agreement with the detailed numerical simulations for both IMA and PMA, as shown in Fig. 1(b). The simulations are carried out within a multiphysics framework [13] using our experimentally benchmarked transmission-line model for SML [14] and the s-LLG model for LBM [15], which considers thermal noise. The details of the simulation setup are discussed in Appendix B.

Here, $I_{0}$ is a parameter that determines the SOT-induced magnetization pinning of the LBM. $I_{0}$ depends on the temperature, geometry and material parameters and is much larger for an IMA as compared to a PMA due to the demagnetization field. $I_{12}$ along the $\mp \hat{n}$ direction causes magnetization pinning along the $\pm \hat{s}$ direction. In the present discussion, the easy axis for PMA is along the $\hat{y}$ direction; hence the pinning occurs along the hard axis. The easy axis of IMA, in principle, can be in any direction on the plane spanned by $\hat{n}$ and $\hat{s}$ and the magnetization pinning along the $\hat{s}$ direction should be described by Eq. (3) with a modified $I_{0}$. However, we set the easy axis along the $\pm \hat{s}$ direction in our IMA simulations for simplicity.

For a given structure, $I_{0}$ can be determined directly from experiments using a characteristic curve similar to that shown in Fig. 1(b) or Fig. 1(c). We provide a simple expression using Eq. (2) and considering easy-axis pinning of a PMA magnet (see Appendix A for the derivation), as given by

$$
I_{0} \approx \frac{6 q}{\hbar} \frac{k_{B} T \alpha_{g}}{\beta}
$$

where $\alpha_{g}$ is the Gilbert damping and $\beta$ is the charge-tospin-current conversion ratio. Equation (4) is reasonably valid up to $\Delta_{B} \approx k_{B} T$ and provides the correct order of magnitude up to several $k_{B} T$ (see Appendix A for details). In this discussion, we consider nanomagnets with very low energy barriers $\left(\leq 1 k_{B} T\right)$, which do not have bistable states. A higher-barrier magnet that exhibits bistable states could, in principle, exhibit effects such as stochastic resonance [31], which is not the subject of the present discussion.

For $\left|I_{12}\right| \gg I_{0}$ in Eq. (3), we have tanh $\left(I_{12} / I_{0}\right) \approx+1$ or -1 when $I_{12}>0$ or $I_{12}<0$, respectively. Hence, $I_{12} \tanh \left(I_{12} / I_{0}\right) \approx\left|I_{12}\right|$. On the other hand, for $\left|I_{12}\right| \ll I_{0}$ in Eq. (3), we have $I_{12} \tanh \left(I_{12} / I_{0}\right) \approx I_{12}^{2} / I_{0}$. Thus, the time average of the voltage in Eq. (1) is given by

$$
\left\langle V_{34}\right\rangle= \begin{cases}\left(\frac{\alpha \xi p_{0} p_{f} R_{B}}{2 I_{0}}\right) I_{12}^{2}, & \text { for }\left|I_{12}\right| \ll I_{0}, \\ \left(\frac{\alpha \xi p_{0} p_{f} R_{B}}{2}\right)\left|I_{12}\right|, & \text { for }\left|I_{12}\right| \gg I_{0} .\end{cases}
$$

Note that $\left\langle V_{34}\right\rangle$ represents the steady-state voltage of the capacitor $C_{L}$ placed between contacts 3 and 4 . The relative position between contacts 3 and 4 along the $\hat{s}$ direction does not affect Eq. (1); however, a shift in the $\hat{n}$ direction creates an offset due to Ohmic drop [20], which should cancel out over averaging in Eq. (5) when an ac $I_{12}$ is applied. For arbitrary $I_{12},\left\langle V_{34}\right\rangle$ is always of the same sign, leading to multiterminal rectification. This observation agrees well with the simulation results for IMA and PMA, as shown in Fig. 1(c). For $\left|I_{12}\right| \ll I_{0},\left\langle V_{34}\right\rangle$ exhibits a parabolic nature [see the enlarged inset of Fig. 1(c)], as suggested by Eq. (5). All of the simulation results presented in this paper are normalized by $I_{0}, V_{0}=I_{0} R_{B}$, and $f_{0}=I_{0} / q$ for the current, voltage, and frequency, respectively. In all of the simulations, $M_{t}=100$, which yields $R_{B}=259 \Omega$. 


\section{THE FREQUENCY BANDWIDTH}

The frequency bandwidth of the multiterminal rectification is limited by a characteristic frequency $f_{c}$ that is determined by the angular-momentum conservation between the spins injected from the SML channel and the spins absorbed by the LBM. We plot the $\left\langle V_{34}\right\rangle$ as a function of the frequency $f$ of the ac $I_{12}=i_{c 0} \sin (2 \pi f t)$ [see Fig. 2(a)] while other parameters are kept constant in our simulations. Note that $\left\langle V_{34}\right\rangle$ is relatively constant in the lowfrequency region and degrades significantly for $f>f_{c}$. We have defined $f_{c}$ as the frequency at which $\left\langle V_{34}\right\rangle$ degrades by an order of magnitude compared to the region in which $\left\langle V_{34}\right\rangle$ vs $f$ is relatively flat.

We observe the time dynamics of $\hat{s} \cdot \vec{m}$ and $V_{34}$ for the two cases indicated with red dots in Fig. 2(a): (I) $f<f_{c}$ and (II) $f>f_{c}$. For the first case, $I_{12}$ is sufficiently slow that the spins injected from the SML channel into the LBM satisfy angular-momentum conservation and $\hat{s} \cdot \vec{m}$ follows $I_{12}$ at the same frequency, as shown in Fig. 2(b). This leads to a rectified voltage $V_{34}$ that charges up the capacitor $C_{L}$ to the steady-state value $\left\langle V_{34}\right\rangle$. The ripples observed in $V_{34}$ are similar to those in conventional rectifiers and become attenuated for increased $C_{L}$. For the latter case, $\hat{s} \cdot \vec{m}$ struggles to follow $I_{12}$ [see Fig. 2(c)] since the spins injected from the SML channel into the LBM are sufficiently fast that they do not satisfy the angular-momentum conservation. $\hat{s} \cdot \vec{m}$ has no correlation with $I_{12}$; as a result, there is no rectification that charges up $C_{L}$ to a steady dc voltage.

We obtain an empirical expression for $f_{c}$ from the detailed s-LLG simulations using a broad range of parameter values, given by

$$
2 \pi f_{c}=\frac{i_{s 0}}{2 q N_{s}}
$$

where the injected spin-current amplitude $i_{s 0}=\beta i_{c 0}, N_{s}=$ $M_{s} \Omega / \mu_{B}$ is the total number of spins in the LBM and $\mu_{B}$ is the Bohr magneton. The functional dependence of $f_{c}$ on
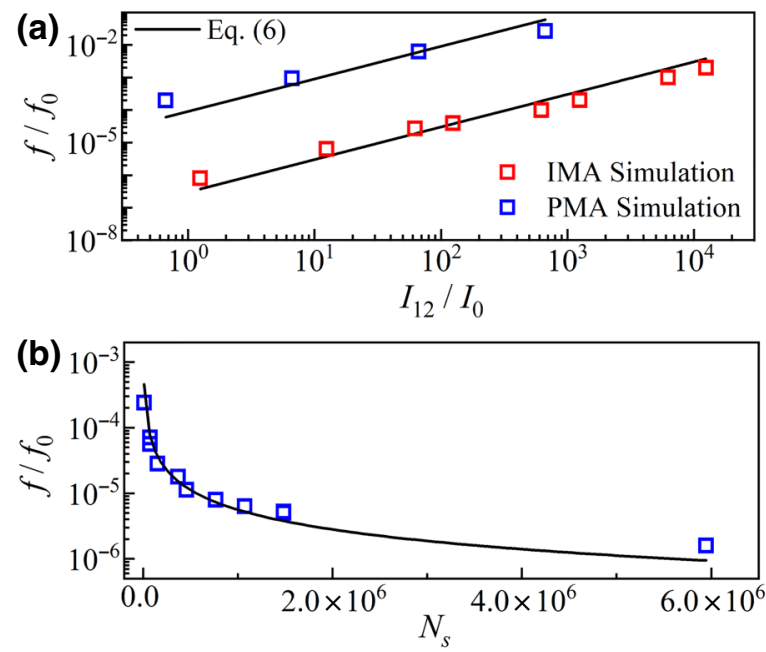

FIG. 3. The characteristic frequency $f_{c}$ is (a) proportional to $i_{c 0}\left(N_{s}=10^{4}\right.$ and $10^{6}$ for PMA and IMA, respectively) and (b) inversely proportional to $N_{s}$. Here, $f_{0}=I_{0} / q$.

$i_{s 0}$ and $N_{s}$ is very similar to the switching delay for stable magnets [32], which also arises from the principles of angular-momentum conservation. Note that Eq. (6) is valid for both IMA and PMA.

We show a comparison between Eq. (6) and the simulation results in Fig. 3. The simulation data points shown in Fig. 3 are extracted from a plot similar to Fig. 2(a). Equation (6) shows good agreement with the simulation for LBMs having IMA with easy-axis pinning and PMA with hard-axis pinning [see Figs. 3(a) and 3(b)]. Figure 3(a) shows that $f_{c}$ scales linearly with $i_{s 0}$. A similar scenario has been reported [33] for a stochastic MTJ oscillator made with a relatively lower-barrier free magnetic layer. According to Eq. (6) and the detailed simulations, the conclusion that $f_{c} \propto i_{s 0}$ seems valid even if $i_{s 0}$ changes by several orders of magnitude. Moreover, Fig. 3(b) shows that the scaling of $f_{c}$ is inversely proportional to $N_{s}$, which

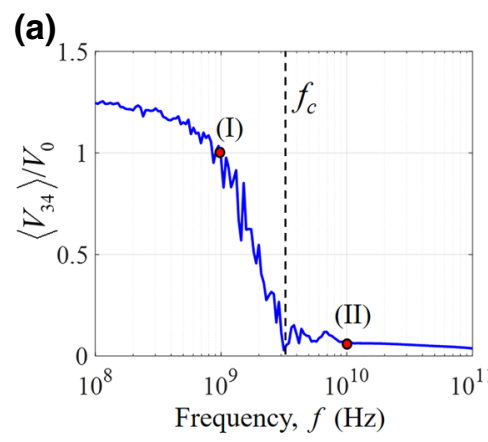

(b)

(I) $f=1 \mathrm{GHz} \quad\left(f<f_{c}\right)$

(c)

(II) $f=10 \mathrm{GHz} \quad\left(f>f_{c}\right)$
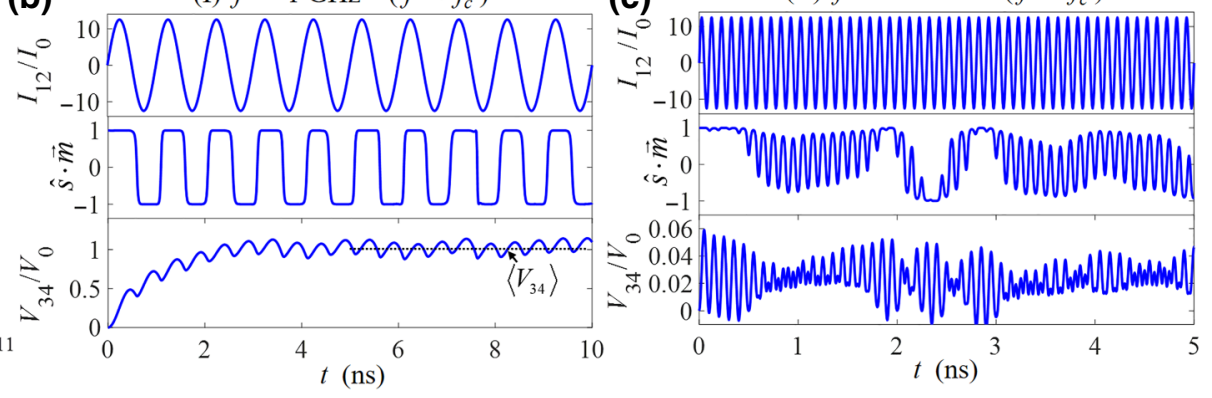

FIG. 2. (a) The rectified voltage $\left\langle V_{34}\right\rangle$ as a function of the input ac frequency $f$, showing the frequency bandwidth $f_{c}$. We observe the time dynamics of the LBM under input current $I_{12}$ for (b) $f<f_{c}$, where, on average, $\hat{s} \cdot \vec{m}$ follows $I_{12}$, leading to rectification and (c) $f>f_{c}$, where $\hat{s} \cdot \vec{m}$ struggles to follow $I_{12}$ and yields no net rectification. The results apply to both IMA and PMA. Here, we consider a $49 \times 61 \times 5 \mathrm{~nm} \mathrm{LBM}$ with $M_{s}=900 \mathrm{emu} / \mathrm{cc} . i_{s 0}=\beta i_{c 0}=2 \mathrm{~mA}$. 
depends only on the $M_{s} \Omega$ value of the magnet and independent of the magnetic anisotropy. Equation (6) could be useful due to the recent interest in LBM-based applications, e.g., stochastic oscillators [33], random-number generators [26,34], probabilistic spin logic [15,35], etc.

\section{APPLICATIONS: RF DETECTION AND ENERGY HARVESTING}

The proposed all-metallic structure can find useful applications such as rf detection and energy harvesting. In this section, we show that the low-barrier nature of the magnet can lead to a very high $\mathrm{rf}$ detection sensitivity without any external bias, comparable to those observed in stateof-the-art technologies under an external bias. We provide a simple model for no-bias sensitivity that provides insight into the design of a high-sensitivity device. This could be of interest for $\mathrm{rf}$ detection from weak sources typically proposed to sense with quantum sensors (see, e.g., Refs. [36] and [37]). We further argue that the proposed structure can extract useful energy from the ambient rf energy, especially from weak sources, where standard technologies may not operate.

It can be seen from Eq. (5) that $\left\langle V_{34}\right\rangle$ scales $\propto \sqrt{P_{\text {in }}}$ when $\max \left(I_{12}\right) \gg I_{0}$, where $P_{\text {in }}=(1 / T) \int_{0}^{T} I_{12}^{2}(t) R_{12} d t$, $T=1 / f$, and $R_{12}$ is the channel resistance. However, for $\max \left(I_{12}\right) \ll I_{0},\left\langle V_{34}\right\rangle$ scales $\propto P_{\text {in }}$, with a constant slope given by

$$
\frac{d\left\langle V_{34}\right\rangle}{d P_{\text {in }}}=\frac{\alpha \xi p_{0} p_{f}}{2} \frac{R_{B}}{I_{0} R_{12}} .
$$

The derivation is given in Appendix C. The quantity in Eq. (7) is often considered as the sensitivity of rf detectors [16-18]. Recently, MTJ diodes with a stable magnet as the free layer and under an external dc-current bias have demonstrated a sensitivity that is several orders of magnitude higher compared to that of state-of-the-art Schottky diodes [16-18]. However, the reported no-bias sensitivity is lower than, or comparable to, that of semiconductor diodes. Equation (7) indicates that the no-external-bias sensitivity can be very high within the all-metallic structure in Fig. 1(a) when it is designed to have very low $I_{0}$, enabling highly sensitive "passive" rf detection. With $\alpha_{g}=0.01$ and $T=300 \mathrm{~K}$, we have $I_{0} \approx 0.37 \mu \mathrm{A} / \beta$ from Eq. (4). For a Permalloy (Py) LBM of dimension of $49 \times$ $61 \times 5 \mathrm{~nm}^{3}$ (see Ref. [27]) and a 2-nm-thick Pt channel, $\beta$ can be approximately 2 , as estimated from the chargeto-spin-conversion ratio reported in Ref. [2], yielding $I_{0} \approx$ $0.18 \mu \mathrm{A}$. Note that $\beta$ can be much higher based on the geometry and the choice of the SML material.

For $\mathrm{Bi}_{2} \mathrm{Se}_{3}$ and $\mathrm{Pt}$, we roughly estimate the sensitivity as 21000 and $860 \mathrm{mV} / \mathrm{mW}$, respectively, assuming a two-dimensional (2D) SML channel of width $w=210 \mathrm{~nm}$ and length $L=500 \mathrm{~nm}$. These estimations were obtained based on Eq. (7) using: (i) $R_{B}=259 \Omega\left(\mathrm{Bi}_{2} \mathrm{Se}_{3}\right)$ and $58 \Omega$ (Pt); (ii) $R_{\mathrm{ch}} \approx 6.5 \mathrm{k} \Omega\left(\mathrm{Bi}_{2} \mathrm{Se}_{3}\right)$ and approximately $3 \mathrm{k} \Omega$ (Pt); (iii) $p_{0} \approx 0.6\left(\mathrm{Bi}_{2} \mathrm{Se}_{3}\right)$ and 0.05 (Pt) (see Ref. [20]); and (iv) $p_{f} \approx 0.5$ [8]. We assume $\xi \approx 1$ and the quoted estimations are lower for higher shunting. $R_{B}$ is estimated using $M_{t}=k_{F} w / \pi$, where $k_{F}=1.5 \mathrm{~nm}^{-1}\left(\mathrm{Bi}_{2} \mathrm{Se}_{3}\right)$ and $6.7 \mathrm{~nm}^{-1}$ (Pt) [20]. The channel resistance is estimated using $R_{\mathrm{ch}}=R_{B}(L+\lambda) / \lambda$ with a mean free path $\lambda$ of $20 \mathrm{~nm}\left(\mathrm{Bi}_{2} \mathrm{Se}_{3}\right.$ [38]) and $10 \mathrm{~nm}(\mathrm{Pt}$ [39]), respectively. We leave more detailed analysis and performance evaluation considering the signal-to-noise ratio for future work.

With proper materials and geometry, it may be possible to extract usable energy from such rectification of $\mathrm{rf}$ signals, especially from weak ambient sources. The dc power $P_{L}=V_{L} I_{L}$ extracted by an arbitrary load $R_{L}$ is limited by the equivalent resistance $R_{34}$ between contacts 3 and 4 . The maximum efficiency of such rf-to-dc power conversion occurs for $\max \left(I_{12}\right) \gg I_{0}$, given by

$$
\eta_{\max }=\frac{P_{L, \max }}{P_{\text {in }}}=\left(\frac{\alpha \xi p_{0} p_{f}}{\sqrt{2} \pi}\right)^{2} \frac{R_{B}}{R_{12}} \frac{R_{B}}{R_{34}} .
$$

The derivation is given in Appendix D. Note that the maximum efficiency is independent of $I_{0}$. Assuming $R_{\text {eq }}=$ $10 R_{B}$ for enhanced $\xi$, we estimate the maximum efficiency to be $0.001 \%$ for $\mathrm{Bi}_{2} \mathrm{Se}_{3}$ and $3 \times 10^{-6} \%$ for Pt, even with $P_{\text {in }}$ in the approximately picowatt range given $I_{0} \leq 0.18 \mu \mathrm{A}$. MTJ diodes have recently demonstrated $\mathrm{rf}$ energy harvesting with similar efficiency [40]; however, the input power was in the microwatt range. Such MTJs should achieve reasonable efficiency at lower input power if the stable free layer is replaced with an LBM.

\section{CONCLUSION}

In conclusion, we predict multiterminal rectification in an all-metallic structure that comprises a spin-orbit material exhibiting SML and a LBM having either in-plane or perpendicular anisotropy (IMA or PMA). The discussion of such multiterminal rectification is limited to the linear response regime of transport and the nonlinearity occurs due to the spin-orbit-torque-driven magnetization dynamics of the LBM. We draw attention to a frequency band of the rectification that can be understood in terms of angular-momentum conservation within the LBM. For a fixed spin-current from the SML channel, the frequency band is same for LBMs with IMA and PMA, as long as they have the same total magnetic moment for a given volume. We further discuss possible applications of the wide-band rectification as a highly sensitive passive $\mathrm{rf}$ detector and in energy harvesting from ambient sources.

\section{ACKNOWLEDGMENTS}

This work was supported by ASCENT, one of six centers in JUMP, a SRC program sponsored by DARPA. 


\section{APPENDIX A: THE AVERAGE MAGNETIZATION OF LOW-BARRIER MAGNETS AND THE MAGNETIZATION-PINNING CURRENT}

This appendix discusses the pinning current of a LBM and derives Eqs. (3) and (4), starting from the steady-state solution of the Fokker-Planck equation.

We start from the steady-state solution of probability distribution from Fokker-Planck equation, assuming a magnet with PMA [see Eq. (4.3) in Ref. [30] ], given by

$$
\rho\left(m_{z}\right)=\frac{1}{\mathrm{Z}} \exp \left[-\frac{\Delta_{B}}{k_{B} T}\left(1-m_{z}^{2}+2\left(\frac{H_{e x t}}{H_{k}}+\frac{i_{s}}{I_{s 0}}\right) m_{z}\right)\right],
$$

where $Z$ is a normalizing factor, $m_{z}$ is the magnetization along the easy axis $\left(\hat{s} \cdot \vec{m}\right.$ in the present discussion), $\Delta_{B}=$ $H_{k} M_{s} \Omega / 2$ is the energy barrier of a magnet with anisotropy field $H_{k}$, saturation magnetization $M_{s}$, and volume $\Omega, k_{B}$ is the Boltzmann constant, $T$ is the temperature, $H_{\text {ext }}$ is the external magnetic field along the easy axis, $i_{s}$ is the $z$-polarized spin current injected into the magnet, and $I_{s 0}$ is the critical spin current for magnetization switching $[20,25]$ for a magnet with PMA, given by

$$
I_{s 0}=\frac{4 q}{\hbar} \Delta_{B} \alpha_{g}
$$

where $\hbar=h /(2 \pi)$ and $\alpha_{g}$ is the Gilbert damping constant.

We consider the case with no external field, i.e., $H_{\text {ext }}=$ 0, which from Eqs. (A1) and (A2) gives

$$
\rho\left(m_{z}\right)=\frac{1}{\mathrm{Z}} \exp \left(-\frac{\Delta_{B}}{k_{B} T}\left(1-m_{z}^{2}\right)-\left(\frac{i_{s}}{\frac{2 q}{\hbar} k_{B} T \alpha_{g}}\right) m_{z}\right) .
$$

We consider a magnet with a very low energy barrier, i.e., $\Delta_{B} / k_{B} T \rightarrow 0$, which in Eq. (A3) yields

$$
\rho\left(m_{z}\right)=\frac{1}{Z} \exp \left(\frac{i_{s}}{\frac{-2 q}{\hbar} k_{B} T \alpha_{g}} m_{z}\right) .
$$

The steady-state average $\left\langle m_{z}\right\rangle$ is defined [see Eq. (2)] as follows:

$$
\left\langle m_{z}\right\rangle=\frac{\int_{\phi=-\pi}^{\phi=\pi} \int_{\theta=0}^{\theta=\pi} m_{z} \rho\left(m_{z}\right) \sin \theta d \theta d \phi}{\int_{\phi=-\pi}^{\phi=\pi} \int_{\theta=0}^{\theta=\pi} \rho\left(m_{z}\right) \sin \theta d \theta d \phi},
$$

with $\left(m_{z}, m_{x}, m_{y}\right) \equiv(\cos \theta, \sin \theta \cos \phi, \sin \theta \sin \phi)$. Combining Eq. (A5) with Eq. (A4), we obtain the long-timeaveraged magnetization $\left\langle m_{z}\right\rangle$ for a very low barrier PMA
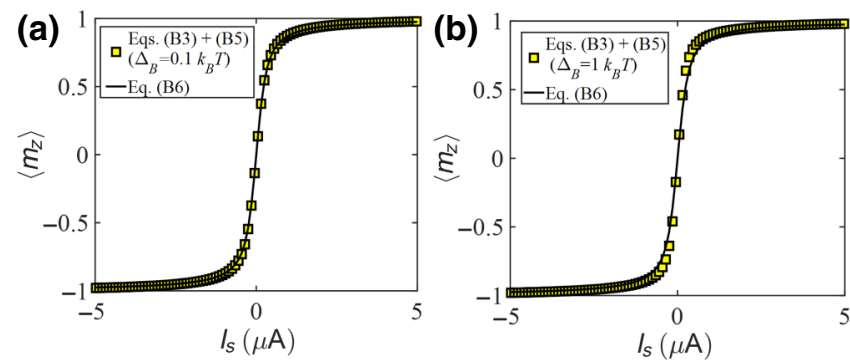

FIG. 4. Comparison of simple expression in Eq. (A6) which assumes $\Delta_{B} \rightarrow 0$ with the numerical calculation from Eqs. (A3) and (A5) for (a) $\Delta_{B}=0.1 k_{B} T$ and (b) $\Delta_{B}=1 k_{B} T$. This comparison indicates that Eq. (A6) is reasonably valid for $0 \leq \Delta_{B} \leq$ $1 k_{B} T$.

without an external magnetic field as follows:

$$
\left\langle m_{z}\right\rangle=\operatorname{coth}\left(\frac{i_{s}}{\frac{2 q}{\hbar} k_{B} T \alpha_{g}}\right)-\frac{\frac{2 q}{\hbar} k_{B} T \alpha_{g}}{i_{s}},
$$

which is a Langevin function $L(x)$ of $x \equiv i_{S} /\left((2 q / \hbar) k_{B} T \alpha_{g}\right)$.

Note that Eq. (A6) is derived assuming that $\Delta_{B} / k_{B} T \rightarrow$ 0 ; however, the expression remains reasonably valid up to $\Delta_{B} \approx k_{B} T$. We compare Eq. (A6) with numerical calculations directly from Eqs. (A3) and (A5) for $\Delta_{B}=0.1 k_{B} T$ [see Fig. 4(a)] and $k_{B} T$ [see Fig. 4(b)] respectively, which shows reasonably good agreement. For $\Delta_{B}>k_{B} T$, the simple expression in Eq. (A6) deviates from Eqs. (A3) and (A5).

For an estimation of the pinning spin current, we can approximate the Langevin function, $L(x) \approx \tanh (x / 3)$, and hence

$$
\left\langle m_{z}\right\rangle \approx \tanh \left(\frac{i_{s}}{\frac{6 q}{\hbar} k_{B} T \alpha_{g}}\right) .
$$

Note that the $i_{s}$ values from SML materials are related to the input charge current $i_{c}$ with a conversion factor $\beta$ given by

$$
i_{s}=\beta i_{c} .
$$

Combination of Eq. (A8) with Eq. (A7) yields

$$
\left\langle m_{z}\right\rangle \approx \tanh \left(\frac{i_{c}}{\frac{6 q k_{B} T \alpha_{g}}{\hbar \beta}}\right) .
$$

Comparison of Eq. (A9) with Eq. (3) yields

$$
I_{0} \approx \frac{6 q k_{B} T \alpha_{g}}{\hbar \beta},
$$

which gives the expression in Eq. (4). 


\section{APPENDIX B: THE SIMULATION SETUP}

This appendix provides the details of the simulation setup in SPICE that is used to analyze the proposed rectifier.

We discretize the structure in Fig. 1(a) into 100 small sections and represent each of the small sections with the corresponding circuit model. Note that each of the nodes in Fig. 5 have two components: the charge $(c)$ and the $z$ component of the spin $(s)$. We connect the charge and spin terminals of the models for all the small sections in a modular fashion using standard circuit rules, as shown in Fig. 5. The models are connected in a series to reconstruct the structure along the length direction. We have two such parallel chains to take into account: the structure along the width direction and the two chains represent the area under the LBM and that under the reference NM, respectively. The SML block with LBM is connected to a s-LLG block, which takes the spin current from the SML block as input and self-consistently solves for $m_{z}$ and feeds back to the SML block.

The contacts $(1,2,3$, and 4$)$ in this discussion are point contacts. The polarization of contacts 1,2 , and 4 is $p_{f}=0$, since they represent normal metals. The polarization of contact 3 is 0.8 , which represents an LBM. We set the total number of modes $M+N$ in the channel to be 100 . We assume that reflection with the spin-flip scattering mechanism is dominant in the channel, i.e., $r_{s 1,2} \gg r, t_{s}$. The scattering rate per unit mode is set to 0.04 per lattice point.

We apply charge-open and spin-ground boundary conditions at the two boundaries given by

$$
\left\{\begin{array}{l}
i_{c} \\
v_{s}
\end{array}\right\}_{L}=\left\{\begin{array}{l}
0 \\
0
\end{array}\right\} \text { and }\left\{\begin{array}{l}
i_{c} \\
v_{s}
\end{array}\right\}_{R}=\left\{\begin{array}{l}
0 \\
0
\end{array}\right\} \text {. }
$$

Here, $i_{c}$ and $v_{s}$ indicate the boundary charge current and the boundary spin voltage. Indexes $L$ and $R$ indicate the left and right boundaries, respectively.

Both the charge and spin terminals of contacts 1 and 2 and the two boundaries of the two parallel model chains

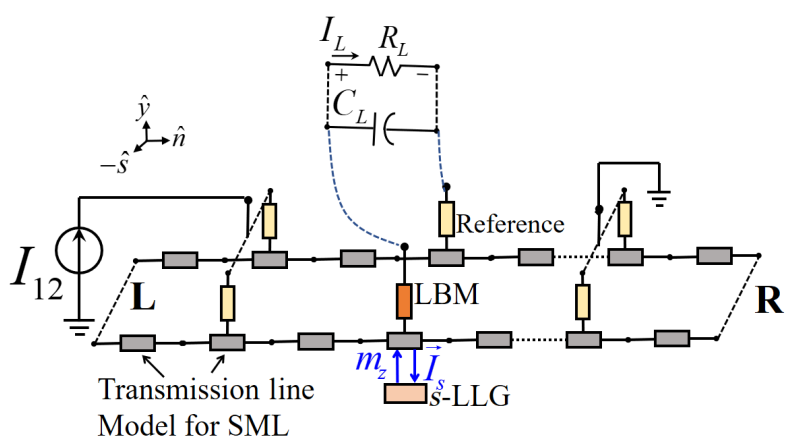

FIG. 5. The spice simulation setup for the structure shown in Fig. 1(a). The SML channel is modeled by connecting a SPICEcompatible transmission-line model [14] in a distributed manner. The LBM is modeled with a s-LLG SPICE model [15]. are connected together. We apply a current $i_{c}$ at the charge terminal of contact 1 and ground the spin terminal to take the spin-relaxation process in the contact into account. We ground both the charge and the spin terminals of contact 2 . The boundary conditions of contacts 1 and 2 are given by the following:

$$
\left\{\begin{array}{l}
i_{c} \\
v_{s}
\end{array}\right\}_{1}=\left\{\begin{array}{c}
i_{c} \\
0
\end{array}\right\} \text { and }\left\{\begin{array}{l}
v_{c} \\
v_{s}
\end{array}\right\}_{2}=\left\{\begin{array}{l}
0 \\
0
\end{array}\right\} .
$$

We place a capacitor $C_{L}$ and load $R_{L}$ across the charge terminals of contacts 3 and 4 . The spin terminals of contacts 3 and 4 are grounded. The boundary conditions of contacts 3 and 4 are given by the following:

$$
\left\{\begin{array}{l}
i_{c} \\
v_{s}
\end{array}\right\}_{3}=\left\{\begin{array}{l}
0 \\
0
\end{array}\right\} \text { and }\left\{\begin{array}{l}
i_{c} \\
v_{s}
\end{array}\right\}_{4}=\left\{\begin{array}{l}
0 \\
0
\end{array}\right\} .
$$

\section{APPENDIX C: SENSITIVITY}

This appendix discusses the detailed derivation of the sensitivity model in Eq. (7).

We start from Eq. (1), with $\vec{m}(t)$ being the instantaneous magnetization of the LBM and calculate the average as follows:

$$
\begin{aligned}
\left\langle V_{34}\right\rangle & =\frac{1}{T} \int_{0}^{T} V_{34} d t \\
& =\frac{1}{T} \frac{\alpha \xi p_{0} p_{f} R_{B}}{2} \int_{0}^{T} \tanh \left(\frac{I_{12}(t)}{I_{0}}\right) I_{12}(t) d t .
\end{aligned}
$$

Note that the timed average of the random fluctuation in the LBM is zero.

We apply an alternating current as input, given by the following:

$$
I_{12}(t)=i_{c 0} \sin \left(\frac{2 \pi t}{T}\right),
$$

The average ac input power applied to the channel with resistance $R_{12}$ is given by the following:

$$
\begin{aligned}
P_{\text {in }} & =\frac{1}{T} \int_{0}^{T} I_{12}^{2}(t) R_{12} d t \\
& =\frac{i_{c 0}^{2} R_{12}}{T} \int_{0}^{T} \sin ^{2}\left(\frac{2 \pi t}{T}\right) d t \\
& =\left(\frac{i_{c 0}}{\sqrt{2}}\right)^{2} R_{12} .
\end{aligned}
$$

\section{Case I: $i_{c 0} \gg I_{0}$}

For $i_{c 0} \gg I_{0}$, we obtain $\tanh \left(I_{12}(t) / I_{0}\right) \approx+1$ when $I_{12}(t)>0$ and $\tanh \left(I_{12}(t) / I_{0}\right) \approx-1$ when $I_{12}(t)<0$. Thus 
we have

$$
\tanh \left(\frac{I_{12}(t)}{I_{0}}\right) I_{12}(t) \approx\left|I_{12}(t)\right|,
$$

and from Eq. (C1), we obtain the following:

$$
\begin{aligned}
\left\langle V_{34}\right\rangle= & \frac{1}{T} \frac{\alpha \xi p_{0} p_{f} R_{B}}{2} \int_{0}^{T}\left|I_{12}(t)\right| d t \\
= & \frac{1}{T} \frac{\alpha \xi p_{0} p_{f} R_{B}}{2} i_{c 0} \int_{0}^{T}\left|\sin \left(\frac{2 \pi t}{T}\right)\right| d t \\
= & \frac{1}{T} \frac{\alpha \xi p_{0} p_{f} R_{B}}{2} i_{c 0} \int_{0}^{T / 2} \sin \left(\frac{2 \pi t}{T}\right) d t \\
& +\frac{1}{T} \frac{\alpha \xi p_{0} p_{f} R_{B}}{2} i_{c 0} \int_{T / 2}^{T}-\sin \left(\frac{2 \pi t}{T}\right) d t \\
= & \frac{\alpha \xi p_{0} p_{f} R_{B}}{2} \frac{2}{\pi} i_{c 0} .
\end{aligned}
$$

We write Eq. (C4) as

$$
\left\langle V_{34}\right\rangle=\frac{\alpha \xi p_{0} p_{f} R_{B}}{\pi} \frac{\sqrt{2}}{\sqrt{R_{12}}} \sqrt{P_{\text {in }}}
$$

and the sensitivity is given by

$$
\frac{d\left\langle V_{34}\right\rangle}{d P_{\text {in }}}=\frac{\alpha \xi p_{0} p_{f} R_{B}}{\pi} \frac{1}{\sqrt{2 R_{12}}} \frac{1}{\sqrt{P_{\text {in }}}} .
$$

The sensitivity for $i_{c 0} \gg I_{0}$ decreases in inverse proportion to $\sqrt{P_{\text {in }}}$. The sensitivity increases for decreasing $P_{\text {in }}$ and eventually saturates to a maximum value for $i_{c 0} \ll I_{0}$.

\section{Case II: $i_{c 0} \ll I_{0}$}

For $i_{c 0} \ll I_{0}$, we obtain $\tanh \left(I_{12}(t) / I_{0}\right) \approx I_{12}(t) / I_{0}$. Thus from Eq. (C1), we obtain the following:

$$
\begin{aligned}
\left\langle V_{34}\right\rangle & =\frac{1}{T} \frac{\alpha \xi p_{0} p_{f} R_{B}}{2 I_{0}} \int_{0}^{T} I_{12}^{2}(t) d t \\
& =\frac{1}{T} \frac{\alpha \xi p_{0} p_{f} R_{B}}{2} \frac{i_{c 0}^{2}}{I_{0}} \int_{0}^{T} \sin ^{2}\left(\frac{2 \pi t}{T}\right) d t \\
& =\frac{\alpha \xi p_{0} p_{f} R_{B}}{2} \frac{\left(i_{c 0} / \sqrt{2}\right)^{2}}{I_{0}} .
\end{aligned}
$$

We write Eq. (C7) as

$$
\left\langle V_{34}\right\rangle=\frac{\alpha \xi p_{0} p_{f} R_{B}}{2 R_{12} I_{0}} P_{\text {in }}
$$

and the sensitivity is given as

$$
\frac{d\left\langle V_{34}\right\rangle}{d P_{\text {in }}}=\frac{\alpha \xi p_{0} p_{f} R_{B}}{2 R_{12} I_{0}},
$$

which gives the maximum sensitivity in Eq. (7).

\section{APPENDIX D: THE POWER-CONVERSION EFFICIENCY}

This appendix discusses the ac-to-dc power-conversion efficiency and provides details of the derivation of Eq. (8).

Under the no-load condition $\left(R_{L} \rightarrow \infty\right)$, we have the open-circuit de voltage from Eq. (C8) for $i_{c 0} \ll I_{0}$,

$$
\left\langle V_{34}\right\rangle=\frac{\alpha \xi p_{0} p_{f} R_{B}}{2 R_{12} I_{0}} P_{\text {in }},
$$

and from Eq. (C5) we know that, for $i_{c 0} \gg I_{0}$,

$$
\left\langle V_{34}\right\rangle=\frac{\alpha \xi p_{0} p_{f} R_{B}}{\pi} \frac{\sqrt{2}}{\sqrt{R_{12}}} \sqrt{P_{\text {in }}} .
$$

Under the short-circuit condition $\left(R_{L} \rightarrow 0\right)$, we have the short-circuit dc current $\left.I_{L}\right|_{R_{L} \rightarrow 0}=\left\langle V_{34}\right\rangle / R_{34}$, where $R_{34}$ is the equivalent resistance between the LBM and the reference NM.

The maximum power transferred to the load is given by the following:

$$
P_{L, \max }=\left.\left.\frac{1}{4} V_{L}\right|_{R_{L} \rightarrow \infty} I_{L}\right|_{R_{L} \rightarrow 0}=\frac{\left\langle V_{34}\right\rangle^{2}}{4 R_{34}},
$$

which yields

$$
\begin{aligned}
P_{L, \max } & =\left(\frac{\alpha \xi p_{0} p_{f} R_{B}}{2 R_{12} I_{0}}\right)^{2} \frac{P_{\text {in }}^{2}}{4 R_{34}} \quad \text { for } i_{c 0} \ll I_{0} \\
& =\left(\frac{\alpha \xi p_{0} p_{f} R_{B}}{\pi}\right)^{2} \frac{P_{\text {in }}}{2 R_{12} R_{34}} \quad \text { for } i_{c 0} \gg I_{0} .
\end{aligned}
$$

The ac-to-dc power-conversion efficiency is given by the following:

$$
\begin{aligned}
\eta=\frac{d P_{L, \max }}{d P_{\text {in }}} & =\left(\frac{\alpha \xi p_{0} p_{f} R_{B}}{2 R_{12} I_{0}}\right)^{2} \frac{P_{\text {in }}}{2 R_{34}} \quad \text { for } i_{c 0} \ll I_{0} \\
& =\left(\frac{\alpha \xi p_{0} p_{f} R_{B}}{\pi}\right)^{2} \frac{1}{2 R_{12} R_{34}} \quad \text { for } i_{c 0} \gg I_{0}
\end{aligned}
$$

Note that $\eta$ increases with input ac power $P_{\text {in }}$ and reaches a maximum when $i_{c 0} \gg I_{0}$ given by the following:

$$
\eta_{\max }=\left(\frac{\alpha \xi p_{0} p_{f} R_{B}}{\pi}\right)^{2} \frac{1}{2 R_{12} R_{34}}
$$

[1] L. Liu, C.-F. Pai, Y. Li, H. W. Tseng, D. C. Ralph, and R. A. Buhrman, Spin-torque switching with the giant spin Hall effect of tantalum, Science 336, 555 (2012). 
[2] L. Liu, O. J. Lee, T. J. Gudmundsen, D. C. Ralph, and R. A. Buhrman, Current-Induced Switching of Perpendicularly Magnetized Magnetic Layers Using Spin Torque from the Spin Hall Effect, Phys. Rev. Lett. 109, 096602 (2012).

[3] C. H. Li, O. M. van't Erve, J. T. Robinson, Y. Liu, L. Li, and B. T. Jonker, Electrical detection of charge-current-induced spin polarization due to spin-momentum locking in $\mathrm{Bi}_{2} \mathrm{Se}_{3}$, Nature Nanotechnol. 9, 20325 (2014).

[4] S. M. Hus, X.-G. Zhang, G. D. Nguyen, W. Ko, A. P. Baddorf, Y. P. Chen, and A.-P. Li, Detection of the SpinChemical Potential in Topological Insulators Using SpinPolarized Four-Probe STM, Phys. Rev. Lett. 119, 137202 (2017).

[5] L. Liu, A. Richardella, I. Garate, Y. Zhu, N. Samarth, and C.-T. Chen, Spin-polarized tunneling study of spinmomentum locking in topological insulators, Phys. Rev. B 91, 235437 (2015).

[6] A. Dankert, J. Geurs, M. V. Kamalakar, S. Charpentier, and S. P. Dash, Room temperature electrical detection of spin polarized currents in topological insulators, Nano Lett. 15, 7976 (2015).

[7] V. T. Pham, L. Vila, G. Zahnd, A. Marty, W. Savero-Torres, M. Jamet, and J.-P. Attané, Ferromagnetic/nonmagnetic nanostructures for the electrical measurement of the spin Hall effect, Nano Lett. 16, 6755 (2016).

[8] J.-H. Lee, H.-J. Kim, J. Chang, S. H. Han, H.-C. Koo, S. Sayed, S. Hong, and S. Datta, Multi-terminal spin valve in a strong Rashba channel exhibiting three resistance states, Sci. Rep. 8, 3397 (2018).

[9] K. M. M. Habib, R. N. Sajjad, and A. W. Ghosh, Chiral Tunneling of Topological States: Towards the Efficient Generation of Spin Current Using Spin-Momentum Locking, Phys. Rev. Lett. 114, 176801 (2015).

[10] J. Tian, S. Hong, I. Miotkowski, S. Datta, and Y. P. Chen, Observation of current-induced, long-lived persistent spin polarization in a topological insulator: A rechargeable spin battery, Sci. Adv. 3, e1602531 (2017).

[11] S. Woo, K. M. Song, H.-S. Han, M.-S. Jung, M.-Y. Im, K.-S. Lee, K. S. Song, P. Fischer, J.-I. Hong, J. W. Choi, B.-C. Min, H. C. Koo, and J. Chang, Spin-orbit torquedriven skyrmion dynamics revealed by time-resolved $\mathrm{x}$-ray microscopy, Nat. Commun. 8, 15573 (2017).

[12] G. Yu, P. Upadhyaya, X. Li, W. Li, S. K. Kim, Y. Fan, K. L. Wong, Y. Tserkovnyak, P. K. Amiri, and K. L. Wang, Room-temperature creation and spin-orbit torque manipulation of skyrmions in thin films with engineered asymmetry, Nano Lett. 16, 1981 (2016).

[13] K. Y. Camsari, S. Ganguly, and S. Datta, Modular approach to spintronics, Sci. Rep. 5, 10571 (2015).

[14] S. Sayed, S. Hong, and S. Datta, Transmission-Line Model for Materials with Spin-Momentum Locking, Phys. Rev. Applied 10, 054044 (2018).

[15] K. Y. Camsari, R. Faria, B. M. Sutton, and S. Datta, Stochastic $p$-Bits for Invertible Logic, Phys. Rev. X 7, 031014 (2017).

[16] S. Miwa, S. Ishibashi, H. Tomita, T. Nozaki, E. Tamura, K. Ando, N. Mizuochi, T. Saruya, H. Kubota, K. Yakushiji, T. Taniguchi, H. Imamura, A. Fukushima, S. Yuasa, and Y. Suzuki, Highly sensitive nanoscale spin-torque diode, Nat. Mater. 13, 50 (2013).
[17] B. Fang, M. Carpentieri, X. Hao, H. Jiang, J. A. Katine, I. N. Krivorotov, B. Ocker, J. Langer, K. L. Wang, B. Zhang, B. Azzerboni, P. K. Amiri, G. Finocchio, and Z. Zeng, Giant spin-torque diode sensitivity in the absence of bias magnetic field, Nat. Commun. 7, 11259 (2016).

[18] L. Zhang, B. Fang, J. Cai, M. Carpentieri, V. Puliafito, F. Garescì, P. K. Amiri, G. Finocchio, and Z. Zeng, Ultrahigh detection sensitivity exceeding $10^{5} \mathrm{~V} / \mathrm{W}$ in spin-torque diode, Appl. Phys. Lett. 113, 102401 (2018).

[19] S. Hong, V. Diep, S. Datta, and Y. P. Chen, Modeling potentiometric measurements in topological insulators including parallel channels, Phys. Rev. B 86, 085131 (2012).

[20] S. Sayed, S. Hong, E. E. Marinero, and S. Datta, Proposal of a single nano-magnet memory device, IEEE Electron Device Lett. 38, 1665 (2017).

[21] S. Sayed, S. Hong, and S. Datta, Multi-terminal spin valve on channels with spin-momentum locking, Sci. Rep. 6, 35658 (2016).

[22] P. Jacquod, R. S. Whitney, J. Meair, and M. Büttiker, Onsager relations in coupled electric, thermoelectric, and spin transport: The tenfold way, Phys. Rev. B 86, 155118 (2012).

[23] J. Kim, C. Jang, X. Wang, J. Paglione, S. Hong, and D. Kim, Electrical detection of surface spin polarization of candidate topological Kondo insulator SmB6, arXiv:1809.04977 [cond-mat.str-el].

[24] P. Li, W. Wu, Y. Wen, C. Zhang, J. Zhang, S. Zhang, Z. Yu, S. A. Yang, A. Manchon, and X.-X. Zhang, Spinmomentum locking and spin-orbit torques in magnetic nano-heterojunctions composed of Weyl semimetal $\mathrm{WTe}_{2}$, Nat. Commun. 9, 3990 (2018).

[25] J. Z. Sun, Spin-current interaction with a monodomain magnetic body: A model study, Phys. Rev. B 62, 570 (2000).

[26] D. Vodenicarevic, N. Locatelli, A. Mizrahi, J. S. Friedman, A. F. Vincent, M. Romera, A. Fukushima, K. Yakushiji, H. Kubota, S. Yuasa, S. Tiwari, J. Grollier, and D. Querlioz, Low-Energy Truly Random Number Generation with Superparamagnetic Tunnel Junctions for Unconventional Computing, Phys. Rev. Applied 8, 054045 (2017).

[27] P. Debashis, R. Faria, K. Y. Camsari, and Z. Chen, Design of stochastic nanomagnets for probabilistic spin logic, IEEE Magn. Lett. 9, 1 (2018).

[28] P. Debashis, R. Faria, K. Y. Camsari, J. Appenzeller, S. Datta, and Z. Chen, in 2016 IEEE International Electron Devices Meeting (IEDM) (2016), pp. 34.3.1.

[29] W. F. Brown, Thermal fluctuations of a single-domain particle, Phys. Rev. 130, 1677 (1963).

[30] W. H. Butler, T. Mewes, C. K. A. Mewes, P. B. Visscher, W. H. Rippard, S. E. Russek, and R. Heindl, Switching distributions for perpendicular spin-torque devices within the macrospin approximation, IEEE Trans. Magn. 48, 4684 (2012).

[31] X. Cheng, C. T. Boone, J. Zhu, and I. N. Krivorotov, Nonadiabatic Stochastic Resonance of a Nanomagnet Excited by Spin Torque, Phys. Rev. Lett. 105, 047202 (2010). 
[32] B. Behin-Aein, A. Sarkar, S. Srinivasan, and S. Datta, Switching energy-delay of all spin logic devices, Appl. Phys. Lett. 98, 123510 (2011).

[33] N. Locatelli, A. Mizrahi, A. Accioly, R. Matsumoto, A. Fukushima, H. Kubota, S. Yuasa, V. Cros, L. G. Pereira, D. Querlioz, J.-V. Kim, and J. Grollier, Noise-Enhanced Synchronization of Stochastic Magnetic Oscillators, Phys. Rev. Applied 2, 034009 (2014).

[34] B. Parks, M. Bapna, J. Igbokwe, H. Almasi, W. Wang, and S. A. Majetich, Superparamagnetic perpendicular magnetic tunnel junctions for true random number generators, AIP Adv. 8, 055903 (2018).

[35] A. Mizrahi, T. Hirtzlin, A. Fukushima, H. Kubota, S. Yuasa, J. Grollier, and D. Querlioz, Neural-like computing with populations of superparamagnetic basis functions, Nat. Commun. 9, 1533 (2018).

[36] M. G. House, T. Kobayashi, B. Weber, S. J. Hile, T. F. Watson, J. van der Heijden, S. Rogge, and M. Y. Simmons, Radio frequency measurements of tunnel couplings and singlet-triplet spin states in $\mathrm{Si}: \mathrm{P}$ quantum dots, Nat. Commun. 6, 8848 (2015).
[37] Mario F. Gely, Marios Kounalakis, Christian Dickel, Jacob Dalle, Rémy Vatré, Brian Baker, Mark D. Jenkins, and Gary A. Steele, Observation and stabilization of photonic Fock states in a hot radio-frequency resonator, Science 363, 1072 (2019).

[38] W. J. Wang, K. H. Gao, and Z. Q. Li, Thickness-dependent transport channels in topological insulator $\mathrm{Bi}_{2} \mathrm{Se}_{3}$ thin films grown by magnetron sputtering, Sci. Rep. 6, 25291 (2016).

[39] G. Fischer, H. Hoffmann, and J. Vancea, Mean free path and density of conductance electrons in platinum determined by the size effect in extremely thin films, Phys. Rev. B 22, 6065 (1980).

[40] B. Fang, M. Carpentieri, S. Louis, V. Tiberkevich, A. Slavin, I. N. Krivorotov, R. Tomasello, A. Giordano, H. Jiang, J. Cai, Y. Fan, Z. Zhang, B. Zhang, J. A. Katine, K. L. Wang, P. K. Amiri, G. Finocchio, and Z. Zeng, Experimental Demonstration of Spintronic Broadband Microwave Detectors and Their Capability for Powering Nanodevices, Phys. Rev. Applied 11, 014022 (2019). 